\title{
Detection and Monitoring of Cancers with Biosensors in Vietnam
}

\author{
A.T. Mai ${ }^{1}$, C.H. Le ${ }^{2}$, P.A. Phan $^{3}$, H.Q. Le ${ }^{4}$, T.Le ${ }^{5}$ and M.S. Packianather ${ }^{6}$ \\ ${ }^{1}$ Biomedical Electronics Center, Hanoi University of Science and Technology, Hanoi, Vietnam \\ ${ }^{2}$ Faculty of Engineering and Science, University of Greenwich, Kent, United Kingdom \\ ${ }^{3}$ The Nuffield Division of Anaesthetics, University of Oxford, Oxford, United Kingdom \\ ${ }^{4}$ Saigon Hi-Tech Park - SHTP, Ho Chi Minh, Vietnam \\ ${ }^{5}$ International University-Vietnam National University, Ho Chi Minh, Vietnam \\ ${ }^{6}$ School of Engineering, Cardiff University, Cardiff, United Kingdom
}

\begin{abstract}
Biosensors are able to provide fast, accurate and reliable detections and monitoring of cancer cells, as well as to determine the effectiveness of anticancer chemotherapy agents in cancer treatments. These have attracted a great attention of research communities, especially in the capabilities of detecting the pathogens, viruses and cancer cells in narrow scale that the conventional apparatus and techniques do not have. This paper presents technologies and applications of biosensors for detections of cancer cells and related diseases, with the focus on the current research and technology development about biosensors in Vietnam, a typical developing country with a very high number of patients diagnosed with cancers in recent years, but having a very low cancer survival rate. The role of biosensors in early detections of diseases, cancer screening, diagnosis and treatment, is more and more important; especially it is estimated that by $2020,60-70 \%$ new cases of cancers and nearly $70 \%$ of cancer deaths will be in economically disadvantaged countries. The paper is also aimed to open channels for the potential $R \& D$ collaborations with partners in Vietnam in the areas of innovative design and development of biosensors in particular and medical technology devices in general.
\end{abstract}

Keywords-Biosensor, DNA sensor, cancer, cancer cell, medical device market, Vietnam

\section{INTRODUCTION}

Biosensors are analytical devices used to detect the presence or concentration of a biological analyte, such as a biomolecule, a biological structure or a microorganism by essentially converting a biological entity such as protein, DNA and RNA into an electrical signal that can be detected and analyzed. Biosensors consist of three parts: a component that recognizes the analyte and produces a signal, a signal transducer, and a reader device. Biosensors can be used to detect emerging cancer biomarkers and to determine drug effectiveness at various target sites; it has the potential to provide a fast and accurate detection, reliable imaging of cancer cells, and monitoring of angiogenesis and cancer metastasis, and the ability to determine the effectiveness of anticancer chemotherapy agents [1]. Today, biosensors play an important role in both diagnosis, monitoring, detection and effective treatments of diseases. This is more important for developing countries with low-income and poor medical services, where the cost of cancer treatments is high com- pared to the income; especially more than half of all cancer cases and nearly $2 / 3$ rd of global cancer deaths occur in developing countries [2].

This paper presents technologies and applications of biosensors for detections of cancer cells and related diseases, with the focus on the current research and technology (R\&D) development about biosensors in Vietnam, a typical developing country with a very high number of patients diagnosed with cancers in recent years, but having a very low cancer survival rate. The potentials for R\&D collaborations with Vietnamese partners are discussed and proposed. The rest of the paper is organized as follows. Section II presents about different types of biosensors, their working principles and applications, and these biosensors are widely studied and successfully developed for medical applications in Vietnam. The discussions are presented in Section III, with the focus on the cancers in Vietnam, and potentials for R\&D collaborations about developments of biosensors for detection, diagnosis, monitoring, and effective treatments of cancers. Finally, Section IV presents a brief conclusion.

\section{BIOSENSORS STUDIED AND SUCCESSFULLY DEVELOPED IN VIETNAM - WORKING AND DETECTION PRINCIPLES}

Fundamentally, a biosensor has a biological element that acts as the sensor and an electronic element or transducer that detects and transmits the signal. The biological element includes enzyme, antibody, living cells and biological tissue; it interacts with the analyte (target) being tested; and the biological response is converted into an electrical signal by the transducer which convert the non-electrical stimulus into electrical signal that can be added, multiplied, divided or amplified. Depending on the types of nonelectrical values that the electronic element can convert, the compatible electronic circuits are designed in a corresponding manner and finally displayed in an easy and friendly way. This leads to different detection types of biosensors, including: Electromechanical, mechanical and optical detections.

\section{A. Electrochemical detection}

Electrochemical biosensors are used to detect cancer biomarkers or cancer cells. The electrochemical biosensors are most preferably studied and developed because the fabrication of these sensors is not difficult; and the readout circuit 
and the operational software can be designed, developed and coded conveniently. However, the detection capability is limited to some applications. The transducer of this biosensor can detect changes in electric properties like capacitance, resistance, current density and/or voltage of the interface between the media solution and the biological membrane that is immobilized on the surface of the transducer.

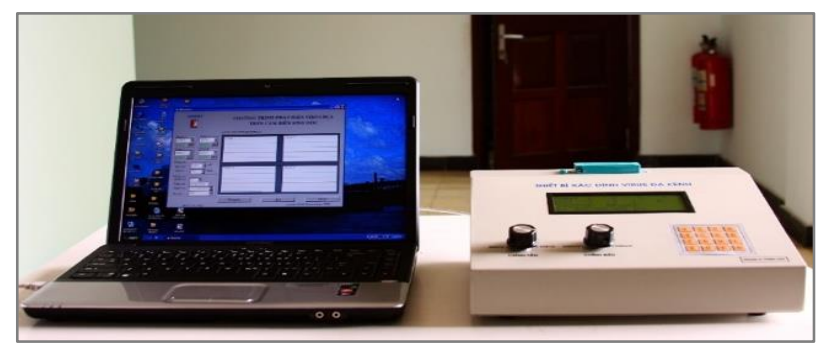

Fig.1 Portable and multichannel equipment for DNA virus detection developed by BIOMAT and NIHE.

The biomaterials and life science research group (BIOMAT) at Hanoi University of Science and Technology (HUST), Vietnam, has performed the conductance measuring resulted by the hybridization between the probe and DNA target for herpes virus detection. The AC reference signals $(10 \mathrm{KHz}, 100 \mathrm{mV})$ produced by the Lock-in Amplifier SR830 generator were applied on two interdigitated electrodes. The output signal was acquired by measuring the voltage drop on two $1 \mathrm{~K} \Omega$ resistances using the $\mathrm{A}$ and $\mathrm{B}$ channels of the Lock-in Amplifier interfaced with a computer. In order to improve the signal to noise ratio, differential measurements were employed. The DNA sensor detected the lowest DNA concentration of $2 \mathrm{nM}$ [19].

Figure 1 presents a portable and multichannel equipment for the DNA virus detection which was successfully developed by BIOMAT and National Institute for Hygiene and Epidemiology (NIHE), Vietnam, based on the electrochemical detection principles. A lot of effort has been made to improve the characteristics of the micro and nanobiosensor by shrinking the size of the transducer [20] or by sensor's surface modification. For example, the DNA Sensor based on the multi-walled carbon nanotubes could detect $0.5 \mathrm{nM}$ of the target DNA samples and the response time of the DNA sensor is approximately 4 minutes [3,4]. Various impact parameters on the hybridization of probe/target DNA such as the hybridization temperature, probe concentration, mismatch target and hybridization time was also investigated [5]. The characteristics of the DNA sensor could also be improved by creating the nanostructure, such as the polypyrrole (PPy) nanowires, on the surface of a transducer.

The electrochemical detection can also be realized by using a cyclic voltammetry, amperometry or potentiostat in both open and narrow spaces. These techniques are generally conducted by changing the applied voltage to the transducer and measuring the change in current or current density that is generated by the biochemical reaction of the biologi- cal elements [6-10]. L.H Nguyen and teams at HUST, Vietnam Academy of Science and Technology, Institute for Tropical Technology (Vietnam), developed electrochemical biosensors using the cyclic voltametry technique for the DNA sensor applications [11]. The technique was also applied for the label-free detection of Carcino-Embryonic Antigens (CEA) [12], and for determination of the residual pesticide in water [13] using the poly (methyl methacrylate) (PMMA) microfluidic device. In addition to the cyclic voltametry, the Electrochemical Impedance Spectroscopy (EIS) was also employed for determination of the human Chorionic Gonadotropin (hCG) using disposable screen-printed electrodes [14].

\section{B. Mechanical detection}

The mechanical detection is normally applied for the transducer of a biosensor that senses with the mechanical properties of the materials. In general, the transducers are developed using the materials that exhibit the piezoelectric effect. Quartz (SiO2) crystal is the most well-known in developing piezoelectric effect-based transducers. Lien T.T.N and teams at HUST, Hue University (Vietnam), Japan Advanced Institute of Science and Technology (Japan) and Korea University (Korea) have modified the surface of a Quartz Crystal Microbalance (QCM) with multi-walled Carbon Nanotube (MWCNTs)-doped polypyrrole (PPy) for the detection of genetically modified organism (GMO) [15]. Regarding the micromachining fabrication, Hien Duy Tong and his teams in the Netherlands and Spain developed the arrays of small nanomechanical resonators with a dual geometry for the DNA detection based on Watson-Crick pairing rules [16]. He also reported the isolation and characterization of the cells by employing the microwell chip [17].

\section{Optical detection}

Compared to the electrochemical detection, there are a few research groups who develop biosensors in which the readout is a photo/optical signal; although this kind of technique has lowest LOD and most reliable. In Vietnam, BIOMAT has been developing the fluorescence base assays based on the optical detection principles. The system consists of the light source, a fluorescence detector; an antigen ready strip that is fixed on the sample holder. The samples (the blood) are deposited on to a strip in which the surface is modified and functionalized with the dye-antibody. Then the specific binding between the antigen (in the sample) and the immobilized antibody (on the strip) will occur. A laser source with the matching wavelength illuminates on the strip and emits the scattering light with corresponding wavelength (photoluminescence in this case). By comparison of the emission density (photoluminescence) given be the sample on the strip and that of the control sample, the analysis will let the operator know if the sample is positive or negative (see Fig.2). BIOMAT has carried out the design and 
realization of the optical filter via deposition of different layers of materials on a surface of a substrate, using the electron beam ion-assisted deposition technique.

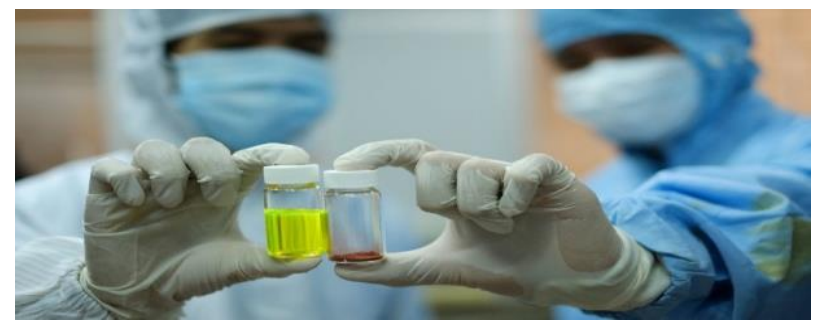

Fig.2 The dye can emit photoluminescence by the UV exciting light.

In addition, BIOMAT has an initial success in creating a new coumarin derivative based biomarker by the combination of a coumarin skeleton that contains 7-diethylamnion as an electron-donating group with the fused polycyclic sulfurcontaining aromatic rings. The dye is soluble in the water or in hybrid solvent; biocompatible; and shows a strong fluorescence at room temperature, with the emission wavelength at $508 \mathrm{~nm}$ (the visible green light, Fig.2) with a very large Stokes' shift (more than $100 \mathrm{~nm}$ ) [17]. The optical sensor based on the microwell was successfully developed by HUST and its partners for detecting the prostate cancer cells which were stained in such a way that they were visible by the fluorescence microscope [18].

\section{Potentials of BIOSENSORS FOR CANCER SCREENING AND DETECTION IN VIETNAM}

Patients diagnosed with cancers have been rapidly increasing in Vietnam in recent years. Many oncology clinics and hospitals in Vietnam were newly established to solve the problems of limited resources and facilities that have not met well the treatment demand. According to recent reports, especially the talk given by National Cancer Hospital ( $\mathrm{K}$ Hospital) ) in Hanoi at The 9th session of the Joint VietnamUS Committee on Scientific and Technological Cooperation (JCM-9) that took place in Ho Chi Minh City on December $2^{\text {nd }} 2015$, Vietnam is now ranked the $12^{\text {th }}$ in the incidence $(2.6 / 100,000)$ and death $(1.0 / 100,000)$ about cancer diseases. Annually, there are nearly 125 thousand people who are diagnosed with the cancers in Viet Nam. K Hospital opened two more additional campuses; and 3 campuses have totally 1,540 beds. The number of patients coming for the cancer examinations in 2014 was 230,388 of which the number coming for the cancer treatments was 42,289.

There have been lots of efforts from research communities in Vietnam who have been working on developments of innovative and cost-effective solutions for early detection and screening as well as monitoring, diagnosis and treatments of cancers. Currently, the conventional methods for early stage cancer screening are mammogram, Magnetic resonance imaging (MRI), Human papillomavirus (HPV) testing, Low-dose helical or spiral computed tomography (CT) scan, Digital rectal examination (DRE), Positron Emission Tomography and Computed Tomography (PET-CT) Scans. The common point of these methods is that, in most cases, the expenditure for the medical test is more than the medical insurance coverage. In addition, the cost of cancer treatments has increased dramatically; fore example, the total direct medical cost for a 5-year treatment course for breast cancer in central Vietnam was estimated at $\$ 975$ per patient [22]. The cancer treatment is expensive for most of the patients; this is a big concern in Vietnam, a typical developing country, with the GDP per capita of 1077.91 USD (2014). Therefore, there is an urgent need for a multidisciplinary approach and cost-effective solutions to improve early detections of cancers and quality of cancer screening services and cancer care, diagnosis and treatments as well as rehabilitation and supportive-care facilities.

\section{DisCUSIONS AND CONCLUSION}

For the last decade, the medical device market in $\mathrm{Vi}$ etnam has been increasing consecutively. The imported medical devices in Vietnam cover more than $90 \%$ of the market. According to the estimation of Espicom [21], the Vietnamese medical device market is expected to increase by $7.0 \%$ in US dollar terms in 2016 . Vietnam is becoming a manufacturing location for medical device companies and exports with the consistent growth, the medical device market will remain one of the strongest performers in the Asia Pacific region. The analysis of Espicom also projects that the market will expand at a 2014-2019 CAGR of $7.9 \%$ in US dollar terms, ranging from $10.9 \%$ for orthopedics' and prosthetics to $4.3 \%$ for other medical devices. The market is forecasted to increase from USD748.9mn in 2014 to USD $1,095.1 \mathrm{mn}$ in 2019. The import performance is expected to improve in 2016, with the economy remaining strong and healthcare expenditure rising each year in line with the expansion of national health insurance coverage and other healthcare programs. In the 12 months to August 2015, imports of medical devices increased by $0.7 \%$ in US dollar terms to USD $662.2 \mathrm{mn}$. For 12 months from August 2014 to August 2015, the exports of medical devices from Vietnam increased by $9.1 \%$ in US dollar terms to USD $766.5 \mathrm{mn}$. With new production companies, such as the United Healthcare plant in Ho Chi Minh City, and continuing collaborations between Vietnamese and foreign companies; it is believed that exports of medical devices will continue to grow in coming years. Table 1 shows the Vietnam's potential market of medical devices in the coming years. In addition, it is estimated that by $2020,60-70 \%$ new cases of cancer and nearly $70 \%$ of cancer deaths will be in economically disadvantaged countries. Therefore, there is an emerg- 
ing need for investments on cost-effective and innovative developments of medical products and services for the cancer scanning, detection, diagnosis and treatments. This opens potential collaborations for $R \& D$ between research institutions in Vietnam and other countries, especially the multi-collaborations among scientist and experts in the areas of medicine, biomaterials, micro-electronics, intelligent computing, biomechanics, micro and nano manufacturing, lab-on-chips, medical image processing, and telehealth.

Table 1 - Projected Medical Device Market, 2014-2019 [21]

\begin{tabular}{ccccc}
\hline Year & $\begin{array}{c}\text { Total } \\
\text { (USD mn) }\end{array}$ & $\begin{array}{c}\text { Per Capita } \\
\text { (USD) }\end{array}$ & $\begin{array}{c}\text { Total } \\
\text { (VND) }\end{array}$ & $\begin{array}{c}\text { Per Capita } \\
\text { (VND mn) }\end{array}$ \\
\hline & & & & \\
\hline 2014 & 748.9 & 8.1 & $15,876,864.8$ & $171,552.8$ \\
\hline 2015 & 781.8 & 8.4 & $16,783,679.5$ & $179,722.5$ \\
\hline 2016 & 836.8 & 8.9 & $17,949,212.8$ & $190,561.8$ \\
\hline 2017 & 913.7 & 9.6 & $19,415,281.9$ & $204,453.8$ \\
\hline 2018 & 999.4 & 10.4 & $21,036,813.9$ & $219,828.3$ \\
\hline 2019 & $1,095.1$ & 11.4 & $22,833,766.9$ & $236,876.8$ \\
\hline
\end{tabular}

Biosensors, especially the DNA sensors and immunosensors, should be promising candidates for R\&D collaborations to develop cost-effective solutions for cancer scanning, detection, diagnosis and treatments, due to its sensitivity, selectivity, response time and reasonable prices. Since cancer screening requires a very Low Limit of Detection (LOD) that may be hard to overcome using electrochemical detection techniques. Meanwhile the optical detection seems to have a much better LOD. Therefore, we propose to innovatively and cost-effectively develop immunosensors via the integration of electrochemical microfluidics and optical sensors, and the use of synthesized biomarkers. Finally, the cost-effective solutions via the use of telehealth and lab-onchips for screening and early detections of diseases should be the core R\&D objectives, especially for the developing and low-income countries. The key to fighting cancer also lies in the early detection and prediction of the decease using the proposed technology.

\section{ACKNOWLEDGEMENT}

Colleagues and PhD students at HUST are acknowledged for their contributions. British Council - Newton Fund is acknowledged for their support.

\section{REFERENCE}

1. Brian B. and Shaker A.M (2010) Biosensors: the new wave in cancer diagnosis. Nanotechnol Sci Appl. 2011: 4: 1-10

2. J. Ferlay et al. (2012) Cancer incidence and mortality worldwide: sources, methods and major patterns in GLOBOCAN 2012, Int. J. Cancer 136 (5): e359-e386

3. P.D Tam et al. (2009) DNA sensor development based on multi-wall carbon nanotubes for label-free influenza virus (type A) detection, Journal of Immunological Methods; 350:118-124

4. T.T Nguyen et al. (2012) Detection of pathogenic microorganisms using biosensor based on multi-walledcarbon nanotubes dispersed in DNA solution, Current Applied Physics; 12(6): 1553-1560
5. D.T Phuong et al. (2009) Impact parameters on hybridization process in detecting influenza virus (type A) using conductimetric-based DNA sensor, Physica E41; 1567-1571

6. D.B Bui and A.T Mai (2013) Electrochemical synthesis of polypyrrole for biosensor application, Int. J. Nanotechnology; 10: 154-163

7. V.T Chuet al. (2013) Polyaniline Nanowires-Based Electrochemical Immunosensor for Label Free Detection of Japanese Encephalitis Virus, Analytical Letters; 46:1229-1240

8. A.T Mai et al. (2014) Highly sensitive DNA sensor based on polypyrrole nanowire, Applied surface science;309:0169-4332

9. T.L Tran et al. (2014) Effective immobilization of DNA for development of polypyrrole nanowires based biosensor, Applied surface science; 314:260-265

10. T.L Tran et al. (2015) In-channel-grown polypyrrole nanowire for the detection of DNA hybridization in an electrochemical microfluidic biosensor, Journal of Nanomaterials; ID 458629

11. L.H Nguyen et al. (2014) Functionalization of reduced graphene oxide by electroactive polymer for biosensing applications, Adv. Nat. Sci.: Nanosci. Nanotechnol; 5:035005

12. V.A Nguyen et al. (2016) Development of a PMMA Electrochemical Microfluidic Device for Carcinoembryonic Antigen Detection, Journal of Electronic Materials; 45:2455-2462

13. H.V. Tran et al. (2012) A label-free electrochemical immunosensor for direct, signal-on and sensitive pesticide detection, Biosensors and Bioelectronics; 31: 62-68

14. Lien TTN et al. (2011) Development of Label-Free Impedimetric Hcg-Immunosensor Using Screen- Printed Electrode. J Biosens Bioelectron; 85(5):2576-80

15. T.N.L Truong et al. (2010) Multi-wall carbon nanotubes (MWCNTs)doped polypyrrole DNA biosensor for label-free detection of genetically modified organisms by QCM and EIS, Talanta; 80:1164-1169.

16. D. Ramos et al. (2009) (2009) Arrays of Dual Nanomechanical Resonators for Selective Biological Detection. Anal. Chem; 81: 2274-2279

17. Q.T Tran et al. (2015) Synthesis of Coumarin Derivatives Based Fluorescent Dye Towards the Detection of Cancer Cell, Vietnam Journal of Chemistry;53(5):641-646

18. J.F Swennenhuis et al. (2015) Self-seeding microwell chip for the isolation and characterization of single cells, Lab Chip;15: 3039-3046

19. P.D Tam et al. (2010) Facile preparation of a DNA sensor for rapid herpes virus detection, Materials Science and Engineering C30; 11451150

20. P.T Tam et al. (2011) Design and fabrication study of DNA sensor based on the integrated electrode arrays, The $3^{\text {rd }}$ international workshop on nanotechnology and application, 2011, pp. 563-566

21. Vietnam Medical Devices Report: www.espicom.com [Access: April 2016]

22. Hoang Lan $\mathrm{N}$ et al. (2013) Cost of treatment for breast cancer in central Vietnam. Glob Health Action; 4 (6): 18872

23. Miriam L.G. et al. (2013) Cancer in developing countries: The next most preventable pandemic. The Global problem of cancer. Critical Reviews in Oncology/Hematology; 88:117-122

Author: Mai Anh Tuan

Institute: Hanoi University of Science and Technology

Address: No. 1, Dai Co Viet Street

City: Hanoi

Country: Vietnam

Email: tuan.maianh@hust.edu.vn 